\title{
Performance Evaluation of T-COFDM under Combined Noise in PLC with Log-Normal Channel Gain using Exact Derived Noise Distributions
}

\author{
Ghanim A. Al-Rubaye, Student Member, IEEE, Charalampos C. Tsimenidis, Senior Member, IEEE, and Martin \\ Johnston,Member, IEEE.
}

\begin{abstract}
In this paper, the performance analyses of the proposed turbo-coded orthogonal frequency division multiplexing (TCOFDM) are investigated over the frequency-selective power-line communication (PLC) with log-normal channel gain based on derived effective complex-valued ratio distributions of the individual and combined noise samples at the zero-forcing (ZF) equalizer output. The effective noise samples are derived in the presence of Nakagami-m background interference (BI) noise, Middleton class A impulsive noise (MCAIN) and their combination. The performance of the soft decoder of the TC has been improved by computing the exact log-likelihood ratio (LLR) using derived distributions, with the derivation of pairwise error probability (PEP) and the average upper-bounds (AUBs). Moreover, the BER degradation in the conventional T-COFDM system has been improved by deriving two clipping thresholds to combat the effect of the non-Gaussian noise, the first one has been derived in the presence of the impulsive noise only modelled by MCAIN model and the second one in the presence of combined Nakagami-m BI noise and MCAIN model. Monte-Carlo simulation results demonstrate significant Bit Error Rate (BER) performance improvements of the proposed T-COFDM system compared to the improved conventional T-COFDM system with a close agreement to the AUBs derivation and analytical BER expression.
\end{abstract}

\section{INTRODUCTION}

Power line communication (PLC) is a technology that utilizes the indoor power line (PL) grid to carry the communication signal along with the AC power signals, such as audio, video and multimedia applications. PLC offers economical communication and can significantly reduce the cost required for the installation of new infrastructure to the system which leads to deployment costs similar to wireless communication [1]. Generally, the PL grid is different to other conventional wired communication channels such as coaxial, fiber-optic or twisted-pair cables. It was originally designed for transmission the electric power at low frequencies, i.e. 50/60 Hz. Hence, PL has hostile properties for low-power high-speed communication signals due to the fluctuating nature of the channel environment, such as reflection points, i.e. multipath fading, attenuation and impulsive noise (IN), which may yield lower data throughput and high Bit Error Rate (BER) degradation [2], [3]. With the advent of advanced technology, people require reliable high-speed data communication in-home and in-building. Therefore, all these factors need to be taken into account to design reliable communication systems.

Noise over PLC channels in the frequency band 1-30 MHz can be divided into background interference (BI) noise and IN [4], [5]. The experimental results presented in [5], [6], demonstrated that the envelope of the BI noise in the time domain follows the Nakagami- $m$ distribution, while the IN can be modelled by the Middleton class A impulsive noise (MCAIN) model [4], [5]. The Nakagami- $m$ BI model and MCAIN model have been the most accurate model for evaluating and analyzing the non-Gaussian noise in PLC systems, and therefore it is adopted in this paper.

The multipath effects and impulsive noise are the dominant performance degradation factors in PLC channels. This is due to the channel frequency selectivity and the high power spectral density (PSD) of IN, which exceeds the PSD of the BI noise by 10-15 dB and may reach $50 \mathrm{~dB}$ with random occurrence rates over a short time duration [4], [7]. Most research in the literature selects orthogonal frequency division multiplexing (OFDM) as an appropriate modulation technique to reduce the errors in data transmission over PLC channels [2]. Therefore, it is adopted in this paper. OFDM is usually used with a cyclic prefix (CP) to achieve highest robustness against frequency-selective fading and to minimize the inter-symbol interference (ISI) that the frequency-selective multipath causes [8]; hence, requiring only a single-tap zero-forcing (ZF) equalizer at the receiver [8], [9]. Generally, OFDM can perform better than single carrier (SC) modulation over PLC channels contaminated by IN, that was because the OFDM spreads the effect of IN over a large number of sub-carriers after fast Fourier transform (FFT) operations at the receiver, which leads to the noise on each subcarrier exhibiting a Gaussian distribution. On the other hand, several non-linear techniques with different degrees of complexity have been developed in the literature to mitigate impulsive noise effects in the time domain to zero and/or clip the incoming samples when exceeding a certain threshold value before the OFDM demodulator. These include clipping, blanking and hybrid techniques [10].

Low-density parity check (LDPC) codes and turbo codes (TCs) with iterative soft decoding algorithm can achieve performance close to the Shannon limit capacity in additive white Gaussian noise (AWGN) channel. The IEEE P1901 draft standard has been chosen the TC as a standard for OFDM system over PLC channels [11] and the TC scheme presented in 3GPP (W-CDMA) and 3GPP2 (CDMA2000) standards [12]. Therefore, we adopted TC in this paper. On the positive side of using TCs against 
LDPC codes, TCs have a significantly low encoder complexity and requires fewer decoder iterations with lower interconnection in the trellis graph than LDPC decoder in the Tanner graph, which has implications for latency. Moreover, a further reduction in the complexity of TC can be achieved by using puncturing. Furthermore, the TCs outperforms the LDPC codes for short codewords length [13]-[15].

On the other hand, The Turbo Convolutional Code (TC) provides superior error correction performance relative to the Turbo Product Code (TPC) over the range of spectral efficiencies, errors correction capability, performance gains are typically $3 \mathrm{~dB}$ or more, high data throughput, reduces the required SNR for the receiving system and the transmit power of other systems causing a reduction of the source of jamming under the consideration by IEEE 802.16.3 [12].

Several states of art over PLC channels are proposed LDPC and turbo codes to improve the performance of SC transmission by utilizing the IN distribution in computation the log-likelihood ratios (LLRs) [16], [17]. Unfortunately, these works are not applicable for the OFDM system due to the fact that the probability density function (PDF) of IN after FFT operations will change. Additionally, ignoring the BI noise and channel multipath effects yield unrealistic results. Moreover, the noise distribution of the MCAIN model that derived in [18, Eq.20] is very complex and inapplicable in a real system. Furthermore, the optimal thresholds for impulsive noise cancellation based on the IN distribution that derived in [10] are very complex to implement even for the simple Bernoulli-Gaussian mixture IN model. Finally, LDPC codes and TCs have been also proposed for multicarrier modulation over PLC channels to improve the BER performance by utilizing frequency diversity [16], [17]. Furthermore, the performance of turbo-codes soft iterative decoding has been improved by proposed a simple LLR computation, the performance of the proposed computation achieves a close result to the performance obtained using a full numerical evaluation of the true LLR and is better than previously proposed approximated solutions in an impulsive channel modelled by $\alpha$-stable IN model [19]. The performance of TCs has been improved by proposed $p$-norm as a new decoding metric over IN channel modelled by systematic- $\alpha$-stable. The proposed $p$-norm metric allows a significant BER improvement compared with the Euclidean distance and the Huber function metrics and its achieves a BER performance close to the optimal decoder metric in severe IN environments and especially when $\alpha \leq 1$ [20]. The performance of TCs in a typical PLC channel contaminated by asynchronous impulsive noise based on actual measurements modelled using Markov chains has been presented in this paper. The Monti-Carlo simulations have been tested based on the code length, the IN energy, the transmission rate and channel interleaving. The simulation results have been determined the sufficient choice of the TC length as a function of the interleaver size, IN energy, channel interleaving and transmission rate. The performance results have been shown the TC can bring much reduction of the transmitted power compared to the convolutional code in the data transmission [21]. The performance of TCs has been improved by using a novel detection scheme at the relay for the physical layer network coding (PLNC) system over multipath PLC channels in the presence of IN modelled by a Gaussian mixture model. The proposed method has been tested by using exact derived IN distributions for the uplink and downlink transmission. The performance results achieved significant improvements in BER compared to the conventional method over all scenarios of IN [22].

Generally, the LLR computations for iterative decoders are highly sensitive to the noise distribution at the equalizer output. For example, the BER performance of the turbo-coded orthogonal frequency division multiplexing (T-COFDM) system utilizes LLRs computed based on the Gaussian noise distribution causes BER degradation in the presence of BI noise, IN or in their combination over multipath PLC channels, due to ignoring the effective noise samples distribution in the frequency domain at the ZF equalizer output.

The major contributions of this paper related to the performance analysis of the T-COFDM system utilizing high order (8192)-quadrature amplitude modulation (8192-QAM) over multipath PLC with Log-Normal Channel Gain in the presence of the individual and combined Nakagami- $m$ type BI noise and IN modeled using MCAIN model are summarized as follows:

- The performance of proposed T-COFDM system has been improved by the following:

-The probability density functions (PDFs) of the effective noise samples at the FFT-OFDM demodulator output and at the ZF equalizer output in the presence of individual and combined Nakagami- $m$ type BI noise and IN modeled using MCAIN model have been derived.

-The performance of iterative soft decoder for the proposed T-COFDM system has been improved by utilizing LLRs computed based on derived effective noise sample distributions at the ZF equalizer output overall scenarios for individual and combined non-Gaussian noise.

-The pairwise error probability (PEP), the average upper-bound (AUB) of $(1,5 / 7,5 / 7)_{8}$ rate- $1 / 3$ TC and three other AUBs have been derived by utilizing derived PDFs over different scenarios of background noise only, impulsive noise only and their combinations to predict the performance of proposed T-COFDM system.

- The performance of conventional T-COFDM system has been improved by deriving simple form clipping threshold expression using Maximum Likelihood (ML) criterion over different scenarios of individual and combined Nakagami- $m$ type BI noise and IN modeled using MCAIN model to compare and evaluate the proposed system with respect to the improved conventional T-COFDM system.

The rest of this paper is organized as follows. The system model is presented in Section 2. The derived PDFs and LLR computation for the proposed T-COFDM system in the presence of different non-Gaussian noise scenarios are illustrated in Section 3. Section IV demonstrate the pairwise error probability and average upper bounds for the proposed T-COFDM system. The threshold optimization and LLR computation for the conventional T-COFDM system are derived in the presence 
of different scenarios of non-Gaussian noise in Section V. Simulation results are presented in Section VI, and finally, Section VII concludes the paper.

\section{T-COFDM SYSTEM OVER PLC CHANNELS}

Parallel concatenated convolutional codes (PCCC) or TCs were invented by Berrou et al. [23] in 1993, as they were found to have excellent coding gain results approaching the Shannon capacity performance through the use of an iterative soft decoding algorithm. The basic block-diagram of the T-COFDM system is shown in Fig. 1.

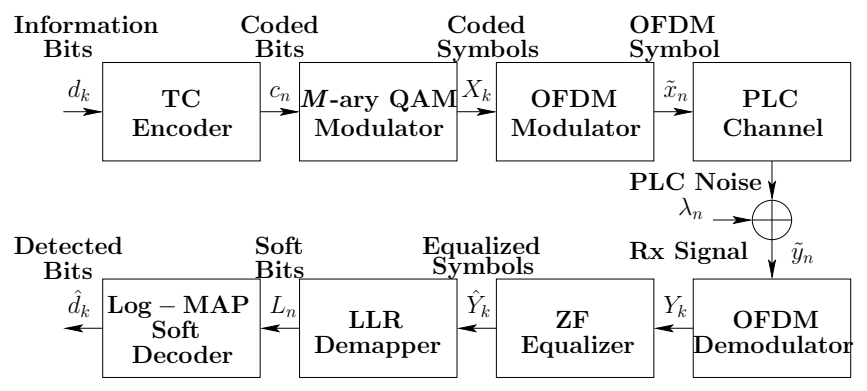

Fig. 1. Block diagram of T-COFDM system over PLC channel using $M$-ary QAM constellation.

The TC can be constructed using two identical parallel Recursive Systematic Convolutional (RSC) codes with Long-Term Evolution (LTE) interleaver between them and "puncturing" stage [23]. The generator matrix uses to generate the code word in this paper can be expressed as $G=(1,5 / 7,5 / 7)$. The information sequence $\mathbf{d}=\left\{d_{0}, d_{1}, \ldots, d_{k_{c}-1}\right\}$ is fed to the first RSC encoder to generate the first parity check bits. The LTE turbo interleavers are highly optimized architectures to provides high performance for extremely fast operations with low latency and enough flexibility to hardware implementations for very high data rates transmission [24], [25]. Therefore, we adopted in this paper. On the second branch, the information sequence $\mathbf{d}$ is interleaved using LTE interleaver and then fed to the second RSC encoder to generate the second parity check bits. Finally, the puncturing mechanism is used to achieve the encoded bits $\mathbf{c}$ for the desired code rate. Subsequently, the bits of the codeword c are then grouped into groups of 13 bits and then mapped unto to the $2^{13}$ symbols of an 8192-QAM constellation to produce the modulated symbols $\mathbf{X}=\mathcal{M}(\mathbf{c})$. Then, the complex base-band OFDM signal $x_{n}$ in the time domain can be obtained using an $N$-points inverse fast Fourier transform (IFFT) as [26].

$$
x_{n}=\frac{1}{\sqrt{N}} \sum_{k=0}^{N-1} X_{k} e^{\frac{j 2 \pi k n}{N}}, n=0,1, \cdots, N-1,
$$

where $X_{k}$ is the complex symbol and $N$ is the number of orthogonal subcarriers. To eliminate ISI between consecutive OFDM symbols in multipath PLC channels, a time-domain CP of length $N_{C P}$ samples, which is designed to exceed the maximum delay spread of the PLC channel $(L)$, is added at the beginning of each OFDM symbol to generate the transmitted symbol $\tilde{\mathbf{x}}=\left[x_{N-N_{C P}}, x_{N-N_{C P}+1}, \ldots, x_{N-1}, x_{0}, x_{1}, \ldots, x_{N-1}\right]$ [2]. Then, $\tilde{\mathbf{x}}$ is sent through the PLC channel.

The gain of the multipath PLC channel of the real and imaginary components has been modelled in the time domain using the log-normal distribution in [27]-[29] and can be expressed as

$$
p_{h}\left(h_{k}^{r}\right)=\frac{1}{\sqrt{2 \pi \sigma^{2}} h_{k}^{r}} e^{\left(-\frac{\left(\ln h_{k}^{r}-\mu\right)^{2}}{2 \sigma^{2}}\right)},
$$

where $h_{k}^{r}>0, r=\{\Re, \Im\}$ represents the real and imaginary components, respectively, $\sigma^{2}$ and $\mu$ are the variance and the mean of the Gaussian random variable, i.e. $\ln h_{k}^{r}$. In this paper, we model the PLC channel gain as log-normal distribution as justified in [27]-[29].

Under perfect synchronization conditions and ISI compensation, the received signal $\tilde{y}_{n}$ in the time domain can be expressed as:

$$
\tilde{y}_{n}=\sum_{i=0}^{L-1} h_{i} \tilde{x}_{n-i}+\lambda_{n}, \quad 0 \leq n \leq N+N_{C P}-1 .
$$

where $\left\{h_{i}\right\}_{i=0}^{L-1}$ are the coefficients of the discrete impulse response of the multipath PLC channel in the time domain and $\boldsymbol{\lambda}=\left[\lambda_{0}, \lambda_{1}, \ldots, \lambda_{\left.N+N_{C P-1}\right]}\right.$ denotes the total non-Gaussian noise samples in the time domain that includes either the BI noise only, the IN only modelled by using MCAIN model or their combination. Hence, $\lambda_{n}$, can be expressed as $\lambda_{n}=b_{n}+i_{n}$, where $b_{n}$ is the BI noise and $i_{n}$ is the IN. The IN is a mixture sources of impulsive noise and background Gaussian noise due to thermal effects in the appliance. 
Assuming perfect time synchronization condition between the transmitter and the receiver, the complex received signal , $\tilde{y}_{n}$, after CP removal can be expressed as $y=\left\{\tilde{y}_{n}\right\}_{0}^{N-1}$ and after the FFT-OFDM demodulator operation can be expressed as

$$
\begin{aligned}
& Y_{k}=\frac{1}{\sqrt{N}} \sum_{n=0}^{N-1} y_{n} e^{\frac{-j 2 \pi n k}{N}}=H_{k} X_{k}+\Lambda_{k}, \\
& \forall k=0,1, \ldots, N-1,
\end{aligned}
$$

where $Y_{k}, H_{k}, X_{k}$ and $\Lambda_{k}$ are the complex received signal in the frequency domain, the complex PLC channel transfer function for the $k$-th sub-carrier, the complex modulated symbols and $\Lambda_{k}=\frac{1}{\sqrt{N}} \sum_{n=0}^{N-1} \lambda_{n} e^{\frac{-j 2 \pi n k}{N}}$ represents the FFT of the total non-Gaussian noise samples $\lambda_{n}$ at the receiver. Thus, the transmitted data symbols can be recovered after the $N$-point FFT operation in (4) by applying single tap ZF equalizer expressed as $\mathrm{ZF}=\frac{1}{H_{k}^{\Re}+j H_{k}^{\Im}}$, yields

$$
\hat{Y}^{\Re}+j \hat{Y}^{\Im}=\frac{Y_{k}^{\Re}+j Y_{k}^{\Im}}{H_{k}^{\Re}+j H_{k}^{\Im}}=X_{k}^{\Re}+j X_{k}^{\Im}+\frac{\Lambda_{k}^{\Re}+j \Lambda_{k}^{\Im}}{H_{k}^{\Re}+j H_{k}^{\Im}},
$$

where $\hat{Y}^{\Re}+j \hat{Y}^{\Im}$ is the received complex-valued equalized signal and $Z_{k}=Z_{k}^{\Re}+j Z_{k}^{\Im}=\frac{\Lambda_{k}^{\Re}+j \Lambda_{k}^{\Im}}{H_{k}^{\Re}+j H_{k}^{\Im}}$ is the complex-valued equalized non-Gaussian noise samples.

\section{EXACT CHANNEL DISTRIBUTION, NOISE DISTRIBUTIONS AND LLR COMPUTATION FOR THE PROPOSED T-COFDM SYSTEM}

\section{A. PLC Channel}

According to the Central Limit Theorem (CLT), the distribution of the real and imaginary components of the channel distribution in (2) will approach a normal distribution after FFT operation in the frequency domain in (4), i.e. $p_{H}\left(H_{k}^{\Re}\right)=$ $\mathcal{N}\left(H_{k}^{\Re}, \mu_{h}=0, \sigma_{h}^{2}\right)$ and $p_{H}\left(H_{k}^{\Im}\right)=\mathcal{N}\left(H_{k}^{\Im}, \mu_{h}=0, \sigma_{h}^{2}\right)$ where $H_{k}^{\Re}$ and $H_{k}^{\Im}$ are zero-mean statistically independent orthogonal Gaussian random variables with variance computed using (2) as

$$
\begin{aligned}
\sigma_{h}^{2} & =\mathbb{E}\left\{\left|h_{k}^{r}\right|^{2}\right\}-\left(\mathbb{E}\left\{\left|h_{k}^{r}\right|\right\}\right)^{2} \\
& =\int_{0}^{\infty} \frac{h_{k}^{r}}{\sqrt{2 \pi \sigma^{2}}} e^{\left(-\frac{\left(\ln h_{k}^{r}-\mu\right)^{2}}{2 \sigma^{2}}\right)} d h_{k}^{r}- \\
& \left(\int_{0}^{\infty} \frac{1}{\sqrt{2 \pi \sigma^{2}} h_{k}^{r}} e^{\left(-\frac{\left(\ln h_{k}^{r}-\mu\right)^{2}}{2 \sigma^{2}}\right)} d h_{k}^{r}\right)^{2} \\
& =\left.\frac{e^{2 \sigma^{2}+2 \mu}}{2} \operatorname{erf}\left(\sqrt{2 \sigma^{2}}\left(-\frac{\mu}{2 \sigma^{2}}+\frac{\log \left(h_{k}^{r}\right)}{2 \sigma^{2}}-1\right)\right)\right|_{0} ^{\infty}- \\
& \left(\left.\frac{e^{\frac{\sigma^{2}}{2}+\mu}}{2} \operatorname{erf}\left(\frac{\sigma}{\sqrt{2}}\left(-\frac{\mu}{\sigma^{2}}+\frac{\log \left(h_{k}^{r}\right)}{\sigma^{2}}-1\right)\right)\right|_{0} ^{\infty}\right)^{2} \\
& =e^{2 \sigma^{2}+2 \mu}-\left(e^{\frac{\sigma^{2}}{2}+\mu}\right)^{2}=e^{2 \mu}\left(e^{2 \sigma^{2}}-e^{\sigma^{2}}\right) .
\end{aligned}
$$

Therefore, the magnitude of the PLC channel in the frequency domain $\left|H_{k}\right|=\sqrt{\left(H_{k}^{\Re}\right)^{2}+\left(H_{k}^{\Im}\right)^{2}}$ exhibits a Rayleigh distribution of two degrees of freedom, i.e. [2]

$$
p_{H}\left(\left|H_{k}\right|\right)=\frac{\left|H_{k}\right|}{\sigma_{H}^{2}} e^{\left(-\frac{\left|H_{k}\right|^{2}}{2 \sigma_{H}^{2}}\right)}, \quad\left|H_{k}\right| \geq 0
$$

and the phase, $\phi_{H_{k}}=\tan ^{-1}\left(\frac{H_{k}^{\Im}}{H_{k}^{\Re}}\right)$, is uniformly distributed as

$$
p_{\phi}\left(\phi_{H_{k}}\right)=\frac{1}{2 \pi} \quad \text { for } \quad-\pi \leq \phi<\pi,
$$

\section{B. Impulsive noise only}

In presence of complex IN only, the non-Gaussian noise, $\lambda_{n}$, in (3) can be expressed as $\lambda_{n}^{\Re}=i_{n}^{\Re}$ and $\lambda_{n}^{\Im}=i_{n}^{\Im}$ representing the real and imaginary IN components at the receiver, respectively, modelled by MCAIN [30], [31]. The distribution of the real and imaginary IN components in the time domain can be expressed as [31]

$$
p_{\lambda}\left(\lambda_{n}^{r}\right)=p_{i}\left(i_{n}^{r}\right)=\sum_{\ell=0}^{\infty} \frac{e^{-A} A^{\ell}}{\ell !} \mathcal{N}\left(i_{n}^{r}, 0, \sigma_{\ell}^{2}\right)
$$


the mathematical expression of this model incorporates both Gaussian noise when $\ell=0$ and IN sources when $\ell \neq 0$ where $\sigma_{\ell}^{2}=\sigma_{w}^{2}\left(1+\frac{\ell}{A \rho}\right)$ denotes the variance associated with the simultaneous emission from $\ell$ noise sources that contribute to the IN, $A$ denotes the average number of impulses during interference time, and $\rho=\frac{\sigma_{w}^{2}}{\sigma_{i}^{2}}$ is the Gaussian-to-impulsive noise power ratio. Different non-linearity based impulsive noise cancellation methods are used in the receiver side in the time domain to limit the amplitude of IN samples before the FFT-OFDM operation, these include clipping, blanking and combining clipping/blanking methods. Moreover, the IN can be suppressed in the Frequency Domain using blanked (nulled) iterative method. Furthermore, one can utilize powerful error correcting codes such as LDPC codes or TCs to mitigate the IN. All the mentioned methods can be used in practice to change the parameter $\rho$ and to improve the BER performance of the system. The FFT operation in (4) will spread the IN over multiple sub-carriers, hence, the noise on each sub-carrier exhibits a Gaussian distribution and can be determined using a statistical approximation. According to the CLT, the distribution of $p_{\Lambda}\left(\Lambda_{k}^{r}\right)$ will approach a normal distribution [5], i.e. $p_{\Lambda}\left(\Lambda_{k}^{r}\right)=p_{I}\left(I_{k}^{r}\right)=\mathcal{N}\left(I_{k}^{r}, \mu_{A}, \sigma_{A}^{2}\right)$ with mean $\mu_{A}=0$ and variance computed using (9) as

$$
\sigma_{A}^{2}=\mathbb{E}\left\{\left(\lambda_{n}^{r}\right)^{2}\right\}-\left(\mathbb{E}\left\{\lambda_{n}^{r}\right\}\right)^{2}=\sum_{\ell=0}^{\infty} \frac{e^{-A} A^{\ell}}{\ell !} \sigma_{w}^{2}\left(\frac{\ell}{A \rho}+1\right) .
$$

Therefore, the magnitude, $\left|\Lambda_{k}\right|=\left|I_{k}\right|=\sqrt{\left(I_{k}^{\Re}\right)^{2}+\left(I_{k}^{\Im}\right)^{2}}$, exhibits a Rayleigh distribution as

$$
p_{\Lambda}\left(\left|\Lambda_{k}\right|\right)=p_{I}\left(\left|I_{k}\right|\right)=\frac{\left|I_{k}\right|}{\sigma_{A}^{2}} e^{\left(\frac{\left|I_{k}\right|^{2}}{2 \sigma_{A}^{2}}\right)},
$$

and the phase $\phi_{\Lambda_{k}}=\phi_{I_{k}}=\tan ^{-1}\left(\frac{I_{k}^{\Im}}{I_{k}^{\Re}}\right)$ exhibits a uniform distribution as

$$
p_{\phi}\left(\phi_{\Lambda_{k}}\right)=\frac{1}{2 \pi} \quad \text { for } \quad-\pi \leq \phi<\pi
$$

Therefore, the complex noise samples at the ZF equalizer output in (5) can be expressed as

$$
Z_{k}=Z_{k}^{\Re}+j Z_{k}^{\Im}=\frac{\left|I_{k}\right| e^{j \phi_{I_{k}}}}{\left|H_{k}\right| e^{j \phi_{H_{k}}}}=\left|\chi_{k}\right| e^{j\left(\phi_{I_{k}}-\phi_{H_{k}}\right)},
$$

where $\phi_{t_{k}}=\phi_{I_{k}}-\phi_{H_{k}}$ is the total phase exhibits a uniform distribution in $[-\pi, \pi)$ as $p_{\phi}\left(\phi_{t_{k}}\right)=\frac{1}{2 \pi}, Z_{k}^{\Re}=\left|\chi_{k}\right| \cos \left(\phi_{t_{k}}\right)$ and $Z_{k}^{\Im}=\left|\chi_{k}\right| \sin \left(\phi_{t_{k}}\right)$ are the real and imaginary components of the equalized noise samples, respectively. Indeed, the PDF of the magnitude part $\left|\chi_{k}\right|=\left|I_{k}\right| \frac{1}{\left|H_{k}\right|}$ can be computed as product of two random variables with Rayleigh distribution and inverse Rayleigh distribution. The distribution of $\frac{1}{\left|H_{k}\right|}$ can be computed by utilizing (7) and by letting $\left|Y_{k}\right|=\frac{1}{\left|H_{k}\right|}$, hence $g^{\prime}=-\frac{1}{\left|H_{k}\right|^{2}}$. The equation $\left|Y_{k}\right|=\frac{1}{\left|H_{k}\right|}$ has a single solution at $\left|H_{k}\right|=\frac{1}{\left|Y_{k}\right|}$ which yields [32]

$$
\begin{aligned}
p_{Y}\left(\left|Y_{k}\right|\right) & =\frac{1}{\left|Y_{k}\right|^{2}} p_{H}\left(\frac{1}{\left|Y_{k}\right|}\right) \\
& =\frac{1}{\sigma_{h}^{2}\left|Y_{k}\right|^{3}} e^{-\frac{1}{2 \sigma_{h}^{2}\left|Y_{k}\right|^{2}}} .
\end{aligned}
$$

The joint PDF between the two random variables $I_{k}$ in (11) and $Y_{k}$ in (14) can be used to compute $\left|\chi_{k}\right|=\left|I_{k}\right|\left|Y_{k}\right|$ as

$$
p_{I, Y}\left(\left|I_{k}\right|,\left|Y_{k}\right|\right)=\frac{\left|I_{k}\right|}{\sigma_{A}^{2} \sigma_{h}^{2}\left|Y_{k}\right|^{3}} e^{-\frac{\left|I_{k}\right|^{2}}{2 \sigma_{A}^{2}}-\frac{1}{2 \sigma_{h}^{2}\left|Y_{k}\right|^{2}}} .
$$

Letting $\left|Y_{k}\right|=\frac{\left|\chi_{k}\right|}{\left|I_{k}\right|}$ in (15) yields

$$
p_{I, Y}\left(\left|I_{k}\right|, \frac{\left|\chi_{k}\right|}{\left|I_{k}\right|}\right)=\frac{\left|I_{k}\right|^{4}}{\sigma_{A}^{2} \sigma_{h}^{2}\left|\chi_{k}\right|^{3}} e^{-\left|I_{k}\right|^{2}\left(\frac{\sigma_{h}^{2}\left|\chi_{k}\right|^{2}+\sigma_{A}^{2}}{2 \sigma_{A}^{2} \sigma_{h}^{2}\left|\chi_{k}\right|^{2}}\right)} .
$$

The PDF of $\left|\chi_{k}\right|$ can be computed by product of two random variables formula in [32], the integration w.r.t $d I_{k}$ yields

$$
\begin{aligned}
& p_{\left|\chi_{k}\right|}\left(\left|\chi_{k}\right|\right)=\int_{0}^{\infty} \frac{1}{\left|I_{k}\right|} p_{I, Y}\left(\left|I_{k}\right|, \frac{\left|\chi_{k}\right|}{\left|I_{k}\right|}\right) d I_{k} \\
& =\int_{0}^{\infty} \frac{\left|I_{k}\right|^{3}}{\sigma_{A}^{2} \sigma_{h}^{2}\left|\chi_{k}\right|^{3}} e^{-\left|I_{k}\right|^{2}\left(\frac{\sigma_{h}^{2}\left|\chi_{k}\right|^{2}+\sigma_{A}^{2}}{2 \sigma_{A}^{2} \sigma_{h}^{2}\left|\chi_{k}\right|^{2}}\right)} d I_{k} \\
& =\frac{2 \sigma_{h}^{2} \sigma_{A}^{2}\left|\chi_{k}\right|}{\left(\sigma_{h}^{2}\left|\chi_{k}\right|^{2}+\sigma_{A}^{2}\right)^{2}}, \text { for }\left(\frac{\sigma_{h}^{2}\left|\chi_{k}\right|^{2}+\sigma_{A}^{2}}{2 \sigma_{A}^{2} \sigma_{h}^{2}\left|\chi_{k}\right|^{2}}\right)^{\Re}>0 .
\end{aligned}
$$


The total phase $\phi_{t_{k}}$ has uniform distribution expressed as $p_{\phi}\left(\phi_{t_{k}}\right)=\frac{1}{2 \pi}$. Thus, the conditional PDF of $p_{Z}\left(Z_{k}^{\Re} \mid \phi_{t_{k}}\right)$ of the real part, $Z_{k}^{\Re}=\left|\chi_{k}\right| \cos \left(\phi_{t_{k}}\right)$, can be expressed as

$$
\begin{aligned}
p_{Z}\left(Z_{k}^{\Re} \mid \phi_{t_{k}}\right) & =\left.\frac{1}{\left|\cos \left(\phi_{t_{k}}\right)\right|} p\left(\left|\chi_{k}\right|\right)\right|_{\left|\chi_{k}\right|=Z_{k}^{\Re} / \cos \left(\phi_{t_{k}}\right)} \\
& =\frac{1}{\left|\cos ^{2}\left(\phi_{t_{k}}\right)\right|} \frac{2 \sigma_{h}^{2} \sigma_{A}^{2} Z_{k}^{\Re}}{\left(\sigma_{h}^{2}\left|\frac{Z_{k}^{\Re}}{\cos \left(\phi_{t_{k}}\right)}\right|^{2}+\sigma_{A}^{2}\right)^{2}},
\end{aligned}
$$

and the joint PDF, $p_{Z, \phi}\left(Z_{k}^{\Re}, \phi_{t_{k}}\right)$, can be expressed as

$$
p_{Z, \phi}\left(Z_{k}^{\Re}, \phi_{t_{k}}\right)=p_{Z}\left(Z_{k}^{\Re} \mid \phi_{t_{k}}\right) p_{\phi}\left(\phi_{t_{k}}\right) .
$$

Hence, the PDF $p\left(Z_{k}^{\Re}\right)$, of the effective noise samples at the ZF equalizer output can be computed as

$$
\begin{aligned}
p_{Z}\left(Z_{k}^{\Re}\right) & =\int_{-\pi}^{\pi} p_{Z, \phi}\left(Z_{k}^{\Re}, \phi_{t_{k}}\right) d \phi_{t_{k}} \\
& =4 \int_{0}^{\pi / 2} \frac{1}{\pi\left|\cos ^{2}\left(\phi_{t_{k}}\right)\right|} \frac{\sigma_{h}^{2} \sigma_{A}^{2} Z_{k}^{\Re}}{\left(\sigma_{h}^{2}\left|\frac{Z_{k}^{\Re}}{\cos \left(\phi_{t_{k}}\right)}\right|^{2}+\sigma_{A}^{2}\right)^{2}} d \phi_{t_{k}},
\end{aligned}
$$

letting $\cos ^{2}\left(\phi_{t_{k}}\right)=t$ gives $d \phi_{t_{k}}=-\frac{d t}{2 \sqrt{t} \sqrt{1-t}}$, then

$$
p_{Z}\left(Z_{k}^{\Re}\right)=2 \int_{0}^{1} \frac{\sigma_{h}^{2} \sigma_{A}^{2} Z_{k}^{\Re} \sqrt{t}}{\pi \sqrt{1-t}\left(\sigma_{h}^{2}\left|Z_{k}^{\Re}\right|^{2}+\sigma_{A}^{2} t\right)^{2}} d t,
$$

integration w.r.t $d t$ yields

$$
p_{Z}\left(Z_{k}^{\Re}\right)=\frac{\sigma_{A}^{2} \sigma_{h}}{2\left(\sigma_{h}^{2}\left|Z_{k}^{\Re}\right|^{2}+\sigma_{A}^{2}\right)^{\frac{3}{2}}} .
$$

It is worth noting that $p_{Z}\left(Z_{k}^{\Im}\right)=p_{Z}\left(Z_{k}^{\Re}\right)$.

\section{Nakagami-m BI noise only}

In the presence of Nakagami- $m$ BI noise only, the non-Gaussian noise, $\lambda_{n}$, in (3) can be expressed as $\lambda_{n}^{\Re}=b_{n}^{\Re}=\tilde{b}_{n} \cos \left(\theta_{n}\right)$ and $\lambda_{n}^{\Im}=b_{n}^{\Im}=\tilde{b}_{n} \sin \left(\theta_{n}\right)$ which represent the real and imaginary components of BI noise at the receiver, respectively. The envelope of the BI noise, $\tilde{b}_{n}$, in the time-domain follows the Nakagami- $m$ distribution [5]. The closed-form distribution of the real and imaginary components, $p_{\lambda}\left(\lambda_{n}^{r}\right)=p_{b}\left(b_{n}^{r}\right)$, for $0<m<1, m \neq \frac{1}{2}$ and $-\infty<b_{n}^{r}<\infty$ have been derived in [6] as

$$
\begin{aligned}
p_{\lambda}\left(\lambda_{n}^{r}\right) & =p_{b}\left(b_{n}^{r}\right)=\frac{e^{-\frac{m\left(b_{n}^{r}\right)^{2}}{\Omega}}}{\sqrt{\pi} \Gamma(m)} \sqrt{\frac{m}{\Omega}}\left[\frac{\Gamma\left(\frac{1}{2}-m\right)}{\Gamma(1-m)} \times\right. \\
& \left(\frac{m\left(b_{n}^{r}\right)^{2}}{\Omega}\right)^{m-\frac{1}{2}}{ }_{1} F_{1}\left(\frac{1}{2}, \frac{1}{2}+m, \frac{m\left(b_{n}^{r}\right)^{2}}{\Omega}\right)+ \\
& \left.\frac{\Gamma\left(m-\frac{1}{2}\right)}{\sqrt{\pi}}{ }_{1} F_{1}\left(1-m, \frac{3}{2}-m, \frac{m\left(b_{n}^{r}\right)^{2}}{\Omega}\right)\right],
\end{aligned}
$$

and for $m=\frac{1}{2}$ as

$$
p_{\lambda}\left(\lambda_{n}^{r}\right)=p_{b}\left(b_{n}^{r}\right)=\frac{1}{\pi} \sqrt{\frac{1}{2 \pi \Omega}} e^{-\frac{\left(b_{n}^{r}\right)^{2}}{4 \Omega}} K_{0}\left(\frac{\left(b_{n}^{r}\right)^{2}}{4 \Omega}\right),
$$

where $m=\left(\mathbb{E}\left\{\tilde{b}_{n}^{2}\right\}\right)^{2} / \mathbb{E}\left\{\left(\tilde{b}_{n}^{2}-\mathbb{E}\left\{\tilde{b}_{n}^{2}\right\}\right)^{2}\right\}$ is the Nakagami- $m$ parameter, $\Omega=\mathbb{E}\left\{\tilde{b}_{n}^{2}\right\}$ is the mean power of the Nakagami$m$ distribution and $\Gamma(\cdot)$ is the Gamma function, ${ }_{1} F_{1}(a ; b ; z)$ is the confluent hypergeometric function expressed as [33, Eq. $\left(9.210^{10}\right)$ ] and $K_{0}(\cdot)$ is the modified Bessel function of the second kind of order zero. The distribution of the noise samples in (23) and (24) at the FFT output has been derived in [34] as $p_{B}\left(B_{k}^{r}\right)=\mathcal{N}\left(B_{k}^{r}, \mu_{b}, \sigma_{b}^{2}\right)=\frac{1}{\sqrt{2 \pi} \sigma_{b}} \exp \left(-\frac{\left(B_{k}^{r}-\mu_{b}\right)^{2}}{2 \sigma_{b}^{2}}\right)$ with mean $\mu_{b}=0$ and the variance $\sigma_{b}^{2}$ computed as [34]

$$
\sigma_{b}^{2}=\frac{\Omega}{2}{ }_{2} F_{1}\left(\frac{3}{2}, \frac{1}{2}, 2,0\right)
$$


where ${ }_{2} F_{1}(a, b ; c ; z)$ is the Gauss hypergeometric function expressed in [33, Eq.(9.14)]. Moreover, the distribution of the effective noise samples at the ZF output for the real and imaginary parts have been derived in [34] as

$$
p_{Z}\left(Z_{k}^{r}\right)=\frac{\sigma_{b}^{2} \sigma_{h}}{2\left(\sigma_{h}^{2}\left|Z_{k}^{r}\right|^{2}+\sigma_{b}^{2}\right)^{\frac{3}{2}}} .
$$

\section{Combined BI noise and IN}

In the presence of the combined Nakagami- $m$ BI noise and MCAIN model, the total non-Gaussian noise, $\lambda_{n}$, in (3) can be expressed as $\lambda_{n}^{\Re}=b_{n}^{\Re}+i_{n}^{\Re}=\tilde{b}_{n} \cos \left(\theta_{n}\right)+i_{n}^{\Re}$ and $\lambda_{n}^{\Im}=b_{n}^{\Im}+i_{n}^{\Im}=\tilde{b}_{n} \sin \left(\theta_{n}\right)+i_{n}^{\Im}$ which denotes the real and imaginary components of the total non-Gaussian noise samples at the receiver, respectively. The complex-valued noise samples after the FFT operation in (4) can be computed by utilizing the sum of two complex random variables as $\xi_{k}=\xi_{k}^{\Re}+j \xi_{k}^{\Im}=$ $\left(B_{k}^{\Re}+I_{k}^{\Re}\right)+j\left(B_{k}^{\Im}+I_{k}^{\Im}\right)$ [32]. The real distribution, $p\left(B_{k}^{\Re}+I_{k}^{\Re}\right)$, and the imaginary distribution, $p\left(B_{k}^{\Im}+I_{k}^{\Im}\right)$, of sum two random variables can be computed using the convolution of their distributions in the probabilistic domain. Hence, the convolution in the probabilistic domain corresponds to product of their characteristic functions in the frequency domain. The characteristic function of the real component of the BI noise, $p_{B}\left(B_{k}^{\Re}\right)$, can be computed as

$$
\Psi_{B}^{\Re}(w)=\mathcal{F}_{t}\left[\frac{1}{\sqrt{2 \pi} \sigma_{b}} e^{-\frac{\left(B_{k}^{\Re}\right)^{2}}{2 \sigma_{b}^{2}}}\right]=e^{-\frac{w^{2} \sigma_{b}^{2}}{2}},
$$

and the characteristic function of the real component of the $\operatorname{MCAIN}, p\left(I_{k}^{\Re}\right)$, can be computed as

$$
\Psi_{I}^{\Re}(w)=\mathcal{F}_{t}\left[\frac{1}{\sqrt{2 \pi} \sigma_{A}} e^{-\frac{\left(I_{K}^{\Re}\right)^{2}}{2 \sigma_{A}^{2}}}\right]=e^{-\frac{w^{2} \sigma_{A}^{2}}{2}},
$$

where $\mathcal{F}_{t}$ represent the Fourier transform operation. The product of their characteristic functions in the frequency domain can be expressed as

$$
\Psi_{B}^{\Re}(w) \Psi_{I}^{\Re}(w)=e^{-\frac{w^{2} \sigma_{b}^{2}}{2}} e^{-\frac{w^{2} \sigma_{A}^{2}}{2}}=e^{-\frac{w^{2}\left(\sigma_{b}^{2}+\sigma_{A}^{2}\right)}{2}} .
$$

Therefore, the PDF of the real part can be computed by taking the inverse Fourier transform of (29) as

$$
\begin{aligned}
p_{\xi}\left(\xi_{k}^{\Re}\right) & =\mathcal{F}_{t}^{-1}\left[\Psi_{B}^{\Re}(w) \Psi_{I}^{\Re}(w)\right]=\mathcal{F}_{t}^{-1}\left[e^{-\frac{w^{2}\left(\sigma_{b}^{2}+\sigma_{A}^{2}\right)}{2}}\right] \\
& =\frac{1}{\sqrt{2 \pi} \sqrt{\sigma_{b}^{2}+\sigma_{A}^{2}}} e^{-\frac{\left(\xi_{k}^{\Re}\right)^{2}}{2\left(\sigma_{b}^{2}+\sigma_{A}^{2}\right)}} .
\end{aligned}
$$

It is worth nothing that $p_{\xi}\left(\xi_{k}^{\Im}\right)=p_{\xi}\left(\xi_{k}^{\Re}\right)$. It can be easily showed that the magnitude of the combined noise, $\left|\xi_{k}\right|=$ $\sqrt{\left(\xi_{k}^{\Re}\right)^{2}+\left(\xi_{k}^{\Im}\right)^{2}}$ exhibits a Rayleigh distribution as $p_{\xi}\left(\left|\xi_{k}\right|\right)=\frac{\left|\xi_{k}\right|}{\sigma_{b}^{2}+\sigma_{A}^{2}} e^{\left(-\frac{\left|\xi_{k}\right|^{2}}{2\left(\sigma_{b}^{2}+\sigma_{A}^{2}\right)}\right)}$ and its phase $\phi_{\xi_{k}}=\tan ^{-1}\left(\frac{\xi_{k}^{\Im}}{\xi_{k}^{\Re}}\right) \operatorname{exhibits}^{-1}$ a uniform distribution as $p_{\phi}\left(\phi_{\xi_{k}}\right)=\frac{1}{2 \pi}$. Thus, the effective noise samples at the ZF equalizer output can be computed by substituting $\left|\xi_{k}\right| e^{j \phi_{\xi_{k}}}$ instead of $\left|I_{k}\right| e^{j \phi_{I_{k}}}$ in (13) and following similar derivation steps as described in (13)-(22) yields

$$
p_{Z}\left(Z_{k}^{r}\right)=\frac{\sigma_{\beta}^{2} \sigma_{h}}{2\left(\sigma_{h}^{2}\left|Z_{k}^{r}\right|^{2}+\sigma_{\beta}^{2}\right)^{\frac{3}{2}}}
$$

where $\sigma_{\beta}^{2}=\sigma_{b}^{2}+\sigma_{A}^{2}$.

\section{E. LLR computation}

The Max-Log-MAP decoding algorithm for Turbo decoder in [35] can be improved by using the modified initial LLR computations based on the derived distributions as

$$
\begin{aligned}
\operatorname{LLR}\left(b_{k}(m)\right) & =\log \frac{\sum_{X_{k} \in \mathcal{M}_{m}^{r}(1)} p_{Z}\left(\hat{Y}_{k}^{r} \mid X_{k}\right)}{\sum_{X_{k} \in \mathcal{M}_{m}^{r}(0)} p_{Z}\left(\hat{Y}_{k}^{r} \mid X_{k}\right)} \\
m & =0,1, \ldots, \log _{2}(M), k=0,1, \ldots, \frac{N-1}{\log _{2}(M)},
\end{aligned}
$$

where the $\mathcal{M}_{m}^{r}(0)$ and $\mathcal{M}_{m}^{r}(1)$ denotes the signal subset of all possible equiprobable symbols of $X_{k}$ whose $\kappa$-th bit is either 0 or 1 in the real and imaginary components. $p_{Z}\left(\hat{Y}_{k}^{r} \mid X_{k}\right)$ represents the the derived distribution in (22) for the case of MCAIN model, (26) for the case of BI noise and (31) for the case of combined BI noise and MCAIN model. 


\section{PAIRWISE ERROR PROBABILITY AND AVERAGE UPPER BOUNDS FOR THE PROPOSED T-COFDM SYSTEM}

The general Symbol Error Rate (SER) expression of the $M$-ary QAM signal for $M>>4$ over PLC channels in the presence of different scenarios of BI noise, MCAIN and their combination can be derived by utilizing the SER of the $\sqrt{M}$-ary pulse amplitude modulation, $\sqrt{M}$-ary PAM, expressed in [9] as

$$
P_{s}^{M-\mathrm{QAM}}=2 P_{s}^{\sqrt{M}-\mathrm{PAM}}-\left(P_{s}^{\sqrt{M}-\mathrm{PAM}}\right)^{2},
$$

the exact solution of (33) is given by [36] as

$$
\begin{aligned}
P_{s}^{M-\mathrm{QAM}} & =2\left(1-\frac{1}{\sqrt{M}}\right)(1-\alpha)-\left(1-\frac{1}{\sqrt{M}}\right)^{2} \\
& \times\left[1-\frac{4}{\pi} \alpha \tan ^{-1}\left(\frac{1}{\alpha}\right)\right],
\end{aligned}
$$

where $\alpha=\sqrt{\frac{K \psi}{K \psi+1}}, \psi=\frac{E_{b} \sigma_{h}^{2}}{\sigma_{R}^{2}}, K=\frac{3 \log _{2}(M)}{2(M-1)}$ and $E_{b}$ is the energy per transmitted bit. Therefore, $P_{s}^{M-Q A M}$, can be determined in the presence of different noise scenarios by substituting $\sigma_{R}^{2}=\sigma_{A}^{2}$ for the case of impulsive noise only, $\sigma_{R}^{2}=\sigma_{b}^{2}$ for the case of background noise only and $\sigma_{R}^{2}=\sigma_{A}^{2}+\sigma_{b}^{2}$ for the case of combined noise.

The AUB of the Turbo Codes is used to determine the bound performance in the high signal-to-noise ratio and beyond simulation capabilities. In order to evaluate the AUB for the PLC channel over different scenarios of non-Gaussian noise, the error probability of decoding the codeword $\mathbf{c}_{2}$ when transmitting the codeword $\mathbf{c}_{1}$ is defined as the PEP or $P_{\nu}$. The $P_{\nu}$ can be computed as [9]

$$
P_{\nu}=\left(P_{s}^{M-\mathrm{QAM}}\right)^{\nu} \sum_{k=0}^{\nu-1}\left(\begin{array}{c}
\nu-1+k \\
k
\end{array}\right)\left(1-P_{s}^{M-\mathrm{QAM}}\right)^{k},
$$

and the union (average) symbol upper bound can be expressed as [37]

$$
P_{s, A U B}^{M-\mathrm{QAM}}=\sum_{\nu} D(\nu) P_{\nu}
$$

and the average bit upper bound can be expressed as

$$
P_{b, A U B}^{M-\mathrm{QAM}}=\frac{P_{s, A U B}^{M-\mathrm{QAM}}}{\log _{2}(M)},
$$

where $D(\nu)$ coefficients are tabulated in [37] for interleaver sizes of 100,1000 and 10000, respectively. Moreover, the AUB can be expressed as given in [38] for different options as

- Option 1:

The exact solution of [38, Eq.(17)] can be computed by integrating it w.r.t $d \phi$ as $P_{2}(\nu) \leq\left(\frac{E_{s}}{N_{o}}\right)^{-\nu} \frac{1}{\pi} \int_{0}^{\pi / 2}[\sin \phi]^{2 \nu} d \phi=$ $\frac{\Gamma\left(\nu+\frac{1}{2}\right)}{2 \sqrt{\pi} \Gamma(\nu+1)}\left(\frac{E_{s}}{N_{o}}\right)^{-\nu}$ where $\frac{E_{s}}{N_{o}}=K \psi$. Hence, the $P_{s}^{\sqrt{M}-\mathrm{PAM}}$ can be computed as

$$
\begin{aligned}
P_{s}^{\sqrt{M}-\mathrm{PAM}} & =2\left(1-\frac{1}{\sqrt{M}}\right) P_{2}(\nu) \\
& =\left(1-\frac{1}{\sqrt{M}}\right) \frac{\Gamma\left(\nu+\frac{1}{2}\right)}{\sqrt{\pi} \Gamma(\nu+1)}[K \psi]^{-\nu},
\end{aligned}
$$

and the SER of $M$-ary QAM signal, $P_{s}^{M-\mathrm{QAM}}$, can be computed by substituting (38) in (33). Then, the symbol AUB can be expressed as

$$
P_{s, A U B}^{M-\mathrm{QAM}} \approx \sum_{\nu} D(\nu) P_{s}^{M-\mathrm{QAM}}
$$

followed by (37) for computing the bit AUB, $P_{b, A U B}^{M-\mathrm{QAM}}$.

- Option 2:

The second option for the upper bounding $P_{2}(\nu)$ can be expressed as given in [38, Eq.(21)] as

$$
P_{2}(\nu) \leq \frac{1}{2}\left[\frac{1}{1+\frac{E_{s}}{N_{0}}}\right]^{\nu} .
$$


The $P_{s}^{\sqrt{M}-\mathrm{PAM}}$ can be computed by utilizing (40) as

$$
\begin{aligned}
P_{s}^{\sqrt{M}-\mathrm{PAM}} & =2\left(1-\frac{1}{\sqrt{M}}\right) P_{2}(\nu) \\
& =\left(1-\frac{1}{\sqrt{M}}\right)\left[\frac{1}{1+K \psi}\right]^{\nu} .
\end{aligned}
$$

$P_{s}^{M-Q A M}$ can be computed by substituting (41) in (33). followed by similar steps that are used in option 1 to compute the $P_{s, A U B}^{M-Q A M}$ in (39) and $P_{b, A U B}^{M-Q A M}$ in (37).

- Option 3:

Another option for upper bounding $P_{2}(v)$ can be expressed as given in [38, Eq.(24)] as

$$
P_{2}(\nu) \leq \frac{1}{2}\left(1-\sqrt{\frac{\frac{E_{s}}{N_{0}}}{1+\frac{E_{s}}{N_{0}}}}\right)\left[\frac{1}{1+\frac{E_{s}}{N_{0}}}\right]^{\nu-1} .
$$

The $P_{s}^{\sqrt{M}-\mathrm{PAM}}$ can be computed by utilizing (42) as

$$
\begin{aligned}
& P_{s}^{\sqrt{M}-\mathrm{PAM}}=2\left(1-\frac{1}{\sqrt{M}}\right) P_{2}(\nu) \\
& =\left(1-\frac{1}{\sqrt{M}}\right)\left[1-\sqrt{\frac{K \psi}{1+K \psi}}\right]\left[\frac{1}{1+K \psi}\right]^{\nu-1},
\end{aligned}
$$

$P_{s}^{M-Q A M}$ can be computed by substituting (43) in (33). Followed by similar steps that are used in option 1 to compute the $P_{s, A U B}^{M-\mathrm{QAM}}$ in (39) and $P_{b, A U B}^{M-\mathrm{QAM}}$ in (37).

\section{CONVENTIONAL T-COFDM SYSTEM}

\section{A. Threshold Optimization}

In order to reduce the high power of the IN in the time domain for the conventional receiver, a clipping non-linearity method can be applied before the OFDM demodulator in Fig. 1, replacing the incoming signal, $\tilde{y}_{n}$ in (3), by the threshold value when it exceeds the clipping threshold $T_{c, M L}^{o p t}$. The output of the clipper can be expressed as [10]

$$
r_{n}=\left\{\begin{array}{cc}
\tilde{y}_{n}, & \left|\tilde{y}_{n}\right| \leq T_{c, M L}^{o p t} \\
T_{c, M L}^{o p t} e^{j \arg \tilde{y}_{n}}, & \text { otherwise }
\end{array}, \quad n=0,1, \cdots, N-1,\right.
$$

where $T_{c, M L}^{o p t}$ is the optimal Maximum Likelihood (ML) threshold. The $T_{c, M L}^{o p t}$ can be computed by using the complex distribution of the MCAIN model in the time domain from (9) as

$$
p\left(\lambda_{n}\right)=\sum_{\ell=0}^{\infty} \frac{e^{-A} A^{\ell}}{\ell !} \frac{\left|\lambda_{n}\right|}{\sigma_{\ell}^{2}} e^{-\frac{\lambda_{n}^{2}}{2 \sigma_{\ell}^{2}}},
$$

where $\left|\lambda_{n}\right|=\sqrt{\left(\lambda_{n}^{\Re}\right)^{2}+\left(\lambda_{n}^{\Im}\right)^{2}}$. The weighted sum of Rayleigh distributions in (45) can be simplified as a mixture of two Rayleigh PDFs, each PDF has a zero mean but with different variances as presented by Spaulding and Middleton in [39], as

$$
p\left(\left|\lambda_{n}\right|\right)=\frac{e^{-A}\left|\lambda_{n}\right|}{\sigma_{w}^{2}} e^{-\frac{\left|\lambda_{n}\right|^{2}}{2 \sigma_{w}^{2}}}+\frac{\left(1-e^{-A}\right)\left|\lambda_{n}\right|}{\zeta^{2}} e^{-\frac{\left|\lambda_{n}\right|^{2}}{2 \zeta^{2}}} .
$$

where $\zeta^{2}=\sigma_{w}^{2}\left(1+\frac{1}{A \rho}\right)$ and $\sigma_{w}^{2}$ is the AWGN variance of coded system uses to define the input Signal-to-Noise Ratio (SNR) as $\mathrm{SNR}=10 \log _{10}\left(\frac{E_{b}}{2 R_{c} \sigma_{w}^{2}}\right)$ where $R_{c}$ is the coding rate. For large number of orthogonal sub-carriers $\mathrm{N}$, the transmitted signal $x_{n}$ in (1) will follow the Gaussian distribution as $\mathcal{N}\left(x_{n}, 0, \sigma_{x}^{2}\right)$. Hence, the complex received signal $\tilde{y}_{n}$ in (3) at the clipper input in (44) has a PDF following the noise PDF according to (46) and can be expressed as

$$
p\left(\left|\tilde{y}_{n}\right|\right)=\frac{e^{-A}\left|\tilde{y}_{n}\right|}{\sigma_{1}^{2}} e^{-\frac{\left|\tilde{y}_{n}\right|^{2}}{2 \sigma_{1}^{2}}}+\frac{\left(1-e^{-A}\right)\left|\tilde{y}_{n}\right|}{\sigma_{2}^{2}} e^{-\frac{\left|\tilde{y}_{n}\right|^{2}}{2 \sigma_{2}^{2}}},
$$

where $\left(1-e^{-A}\right)$ denotes the probability of impulsive occurrence, $\sigma_{1}^{2}$ denotes the variance of the received signal in the case of free impulsive and $\sigma_{2}^{2}$ denotes the variance of the received signal in the case of impact impulsive. Using the ML [9] based on combination criterion, the decision rule based on (47) can be expressed as

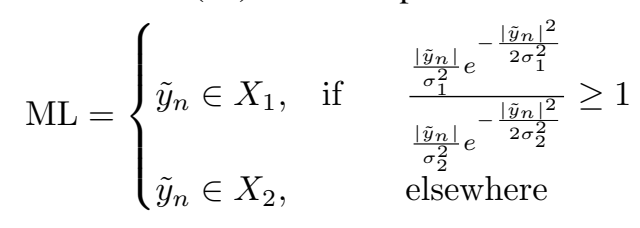


The decision rule is exactly equivalent to

$$
\mathrm{ML}=\left\{\begin{array}{lcc}
\tilde{y}_{n} \in X_{1}, & \text { if } & \tilde{y}_{n} \leq T_{c, M L}^{o p t} \\
\tilde{y}_{n} \in X_{2}, & \text { elsewhere }
\end{array}\right.
$$

where $T_{c, M L}^{o p t}$ is the ML criterion threshold which can be computed based on (48) as [9], [40]

$$
\begin{aligned}
\frac{\left|T_{c, M L}^{o p t}\right|}{\sigma_{1}^{2}} e^{-\frac{\left|T_{c, M L}^{o p t}\right|^{2}}{2 \sigma_{1}^{2}}} & =\frac{\left|T_{c, M L}^{o p t}\right|}{\sigma_{2}^{2}} e^{-\frac{\left|T_{c, M L}^{o p t}\right|^{2}}{2 \sigma_{2}^{2}}}, \\
\left|T_{c, M L}^{o p t}\right| & =\sqrt{\frac{2 \sigma_{2}^{2} \sigma_{1}^{2}}{\sigma_{2}^{2}-\sigma_{1}^{2}} \ln \left(\frac{\sigma_{2}^{2}}{\sigma_{1}^{2}}\right)} .
\end{aligned}
$$

Thus, in the presence of MCAIN only, letting $\sigma_{1}^{2}=\frac{\mathbb{E}\left\{|h|^{2}\right\} \mathbb{E}\left\{|x|^{2}\right\}}{2}+\sigma_{w}^{2}$ and $\sigma_{2}^{2}=\frac{\mathbb{E}\left\{|h|^{2}\right\} \mathbb{E}\left\{|x|^{2}\right\}}{2}+\zeta^{2}$. The optimal threshold can be computed by utilizing (50) and after some simplifications yields

$$
\begin{aligned}
&\left|T_{c, M L}^{o p t}\right|= \sqrt{2 A \rho \sigma_{w}^{2}(\Psi+1)\left(\Psi+\frac{1}{A \rho}+1\right)} \times \\
& \sqrt{\ln \left(1+\frac{1}{A \rho(\Psi+1)}\right)} .
\end{aligned}
$$

While, in case of combined BI noise and MCAIN, letting $\sigma_{1}^{2}=\frac{\mathbb{E}\left\{|h|^{2}\right\} \mathbb{E}\left\{|x|^{2}\right\}}{2}+\sigma_{b}^{2}+\sigma_{w}^{2}$ and $\sigma_{2}^{2}=\frac{\mathbb{E}\left\{|h|^{2}\right\} \mathbb{E}\left\{|x|^{2}\right\}}{2}+\sigma_{b}^{2}+\zeta^{2}$. The clipping threshold can be computed by utilizing (50) and after some simplifications yields

$$
\begin{aligned}
\left|T_{c, M L}^{o p t}\right| & =\sqrt{2 A \rho \sigma_{w}^{2}\left(\Psi+\frac{\sigma_{b}^{2}}{\sigma_{w}^{2}}+1\right)\left(\Psi+\frac{\sigma_{b}^{2}}{\sigma_{w}^{2}}+\frac{1}{A \rho}+1\right)} \\
& \times \sqrt{\ln \left(\frac{1}{A \rho\left(\Psi+\frac{\sigma_{b}^{2}}{\sigma_{w}^{2}}+1\right)}+1\right)} .
\end{aligned}
$$

where $\Psi=\frac{\mathbb{E}\left\{|h|^{2}\right\} \mathbb{E}\left\{|x|^{2}\right\}}{2 \sigma_{w}^{2}}$. Next, the output of the clipper $r_{n}$ in (44) is OFDM demodulated and then equalized by ZF equalizer.

\section{B. LLR Computation}

The LLR of Turbo decoder for the conventional system can be computed based on Gaussian distribution as

$$
\begin{aligned}
\operatorname{LLR}\left(b_{k}(m)\right)=\log \frac{\sum_{X_{k} \in \mathcal{M}_{m}^{r}(1)} e^{-\left(\frac{\left|\hat{Y}_{k}^{r}-X_{k}\right|^{2}}{\sigma_{w}^{2}}\right)}}{\sum_{X_{k} \in \mathcal{M}_{m}^{r}(0)} e^{-\left(\frac{\left|\hat{Y}_{k}^{r}-X_{k}\right|^{2}}{\sigma_{w}^{2}}\right)}} \\
m=0,1, \ldots, \log _{2}(M), k=0,1, \ldots, \frac{N-1}{\log _{2}(M)} .
\end{aligned}
$$

\section{SIMULATION RESULTS}

\section{A. Histogram plot}

In this section, to verify the validity of the derived PDFs, a comparison of the histogram plots of the real part between the empirical and theoretical noise PDFs in (22), (26) and (31) in the presence of IN, BI noise and their combination over PLC with log-normal channel gain are shown in Fig.(2). The channel is generated for the parameters $\mu=0$ and $\sigma^{2}=$ 0.05 at SNR equal $10 \mathrm{~dB}$, the $\sigma_{H}^{2}$ computed as 0.0539 using (6). The BI noise and MCAIN model parameters are set as $m=0.7, \Omega=1, A=0.01, \ell=0-100$ and $\rho=0.02$. The variances are computed using the system parameters can be expressed as $\sigma_{A}^{2}=2.55, \sigma_{b}^{2}=0.5$ and $\sigma_{\beta}^{2}=3.05$ for MCAIN, BI noise and their combination, respectively. It is worth noting that the closed-form PDFs closely match with their corresponding empirically obtained distributions using Monte-Carlo simulations. The mean squared error (MSE) between the empirical and theoretical noise distributions can be computed using $\mathrm{MSE}=\frac{1}{N} \sum_{k=0}^{N-1}\left(\hat{Z}_{k}^{\Re}-Z_{k}^{\Re}\right)^{2}$ as $1.5228 \times 10^{-9}, 4.8393 \times 10^{-10}$ and $4.9128 \times 10^{-9}$ for IN, BI noise and and their combination, respectively. Similar conclusions and MSE results were observed when the imaginary part was used. We can conclude that due to orthogonality, the real and imaginary components of the noise exhibit identical statistical behaviour. 


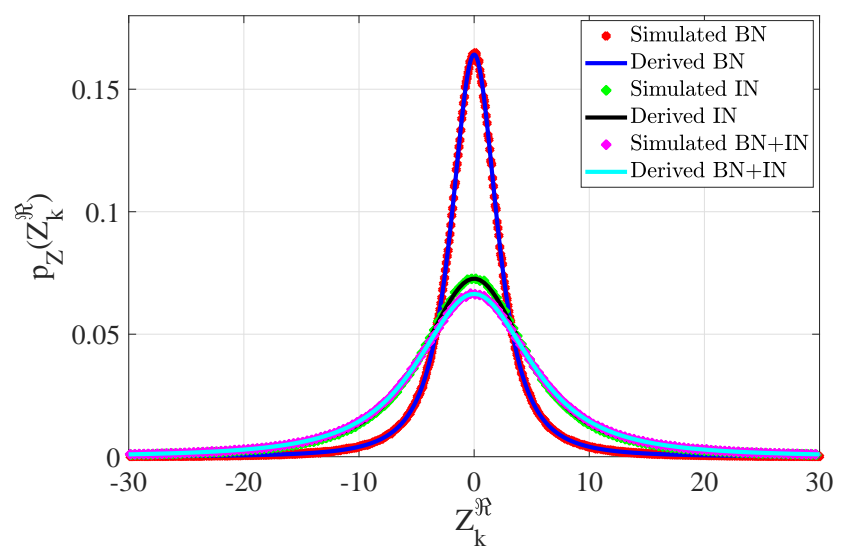

Fig. 2. Histogram plot of different scenarios of non-Gaussian noise over PLC with log-normal channel gain based on the derived PDFs.

\section{B. Performance of proposed vs conventional T-COFDM system}

In order to assess the performance of the proposed T-COFDM system over the PLC channel over different scenarios of individual and combined BI noise and MCAIN model, the derived PDFs are utilized. The simulation parameters were set as follows, the number of sub-carriers was set as $\mathrm{N}=1024$ and $\mathrm{CP}=256$, the constellation size was set as 8192-QAM. The rate- $\frac{1}{3}$ TC has been constructed using the generator $(1,5 / 7,5 / 7)_{8}$ with 50 iterations and LLR computed based on the derived PDFs. The BER performances are investigated over PLC with log-normal channel gain of six paths. The performance of the proposed T-COFDM system is compared against the conventional T-COFDM system, in which the clipping non-linearity impulsive noise mitigation method in (51) and (52) are utilized, and the LLR computed based on the Gaussian distribution. Both systems performance are compared against the uncoded OFDM (UOFDM) system over PLC channel in the presence of different scenarios of non-Gaussian noise.

In Fig. 3-a, we illustrate the BER performance comparison between theoretical AUB bounds and Monte Carlo simulation results in the presence of BI noise only with $m=0.7$. The LLR has been computed by utilizing (32) based on the exact noise PDF in (26) compared to the conventional system that utilizes the LLR computed based on the Gaussian distribution in (53). The BER performance of both systems are compared to the uncoded Monte Carlo simulation and the derived theoretical BER computed based on (34) after division by $\log _{2}(8192)$ for $\sigma_{R}^{2}=\sigma_{b}^{2}$. It can be seen from the figure that the proposed receiver outperforms the conventional receiver by $4.75 \mathrm{~dB}$ at $\mathrm{BER}=10^{-5}$ and gives about $34.4 \mathrm{~dB}$ coding gain (CG) compared to 29.65 $\mathrm{dB}$ in the case of the conventional receiver. Moreover, the AUB correctly predicts the system performance and gives closely matching to the proposed system performance. Furthermore, the derived theoretical performance of UOFDM system exhibits close matching to Monte-Carlo simulation results.

Fig. 3-b, shows the BER performance of the proposed T-COFDM system versus the conventional T-COFDM system in the presence of IN only modelled by using MCAIN model. The LLR has been computed using (32) based on the exact noise PDF in (22) for the proposed system. While the conventional system has been utilized the clipping IN mitigation method based on the optimal derived clipping threshold in (51) and LLR computed based on Gaussian distribution in (53). The impulsive noise parameters are set as $\ell=0-100, A=10^{-2}$ and $\rho=10^{-1}, 10^{-3}$. Both systems performance is compared to the derived theoretical BER performance of UOFDM system in (34) divided over $\log _{2}(8196)$ for $\sigma_{R}^{2}=\sigma_{A}^{2}$. It can be seen from the figure that the proposed receiver outperforms the conventional receiver in all cases, and the AUBs closely matching to the performance of the proposed system. For example, the proposed and conventional systems achieves approximately CGs as $33.25,29.4 \mathrm{~dB}$ and $33.6,29.9 \mathrm{~dB}$ for $\rho=10^{-1}, 10^{-3}$, respectively, at $\mathrm{BER}=10^{-5}$. Furthermore, the derived theoretical performance of UOFDM system exhibits close matching to Monte-Carlo simulation results.

Fig. 4-a, shows the BER performance of both systems in the presence of combined BI noise and MCAIN model. The proposed system utilizes the LLR in (32) computed based on the derived PDF in (31). While the conventional receiver utilizes the clipping IN cancellation based on the derived clipping threshold in (52) and LLRs computed based on the Gaussian distribution in (53). The IN parameters are taken as $m=0.7, \ell=0-100$, for constant $A=10^{-2}$ and changing $\rho$ as $\rho=10^{-1}, 10^{-3}$. Both systems performance are compared to the derived theoretical BER for UOFDM system in (34) divided over $\log _{2}(8196)$ for $\sigma_{R}^{2}=\sigma_{b}^{2}+\sigma_{A}^{2}$. It can be seen from the figure that the BER performance of both systems are worse than the performance in the presence of MCAIN only and the performance depends on changing $\rho$. However, when $\rho$ decreases, $\sigma_{A}^{2}$ will increase, which implies that the noise variance $\sigma_{\beta}^{2}=\sigma_{b}^{2}+\sigma_{A}^{2}$ becomes stronger, therefore, the performance becomes worse. Moreover, the performance of the proposed receiver is more robust against the combined noise than the conventional receiver with AUBs closely match to the proposed system simulation results. For example, the CGs of both systems are about $33.1,28.5 \mathrm{~dB}$ and $33.4,29 \mathrm{~dB}$ for $\rho=10^{-1}, 10^{-3}$ for the case of the proposed and the conventional receiver, respectively, at 


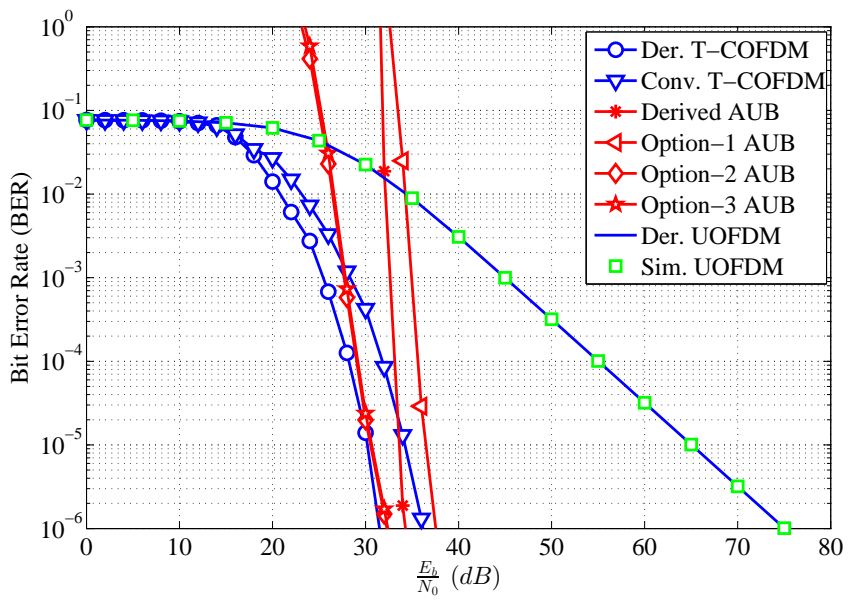

(a) BI noise only with $m=0.7$.

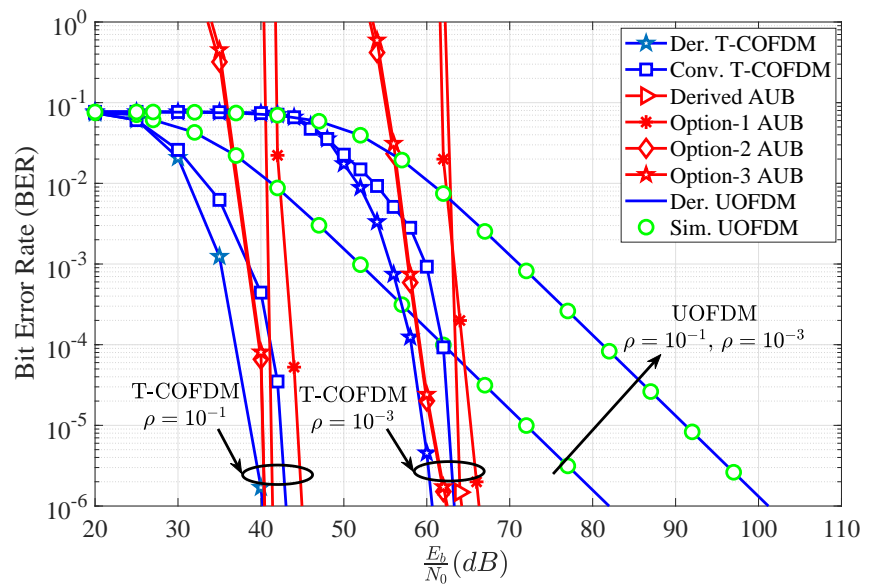

(b) IN only with $A=10^{-2}$ and $\rho=10^{-1}, 10^{-3}$.

Fig. 3. Performance of proposed T-COFDM system versus conventional T-COFDM system utilizing 8192-QAM constellation over PLC channel with lognormal channel gain.

TABLE I

RUNNING TIME IN SECOND.

\begin{tabular}{|c|c|c|c|c|c|}
\hline $\begin{array}{c}\rho=10^{-1} \\
\frac{E_{b}}{N_{0}}(\mathrm{~dB})\end{array}$ & Proposed system & Conventional system & $\begin{array}{c}\rho=10^{-3} \\
\frac{E_{b}}{N_{0}}(\mathrm{~dB})\end{array}$ & Proposed system & Conventional system \\
\hline 20 & 1112.553 & 1254.528 & 40 & 1140.150 & 1295.441 \\
\hline 30 & 634.422 & 880.099 & 50 & 708.346 & 915.116 \\
\hline 40 & 212.982 & 519.376 & 60 & 297.120 & 604.488 \\
\hline
\end{tabular}

$\mathrm{BER}=10^{-5}$.

Fig. 4-b shows the BER performance result of both systems in the presence of combined BI noise and IN modelled by MCAIN model. The noise parameters are taken as $m=0.7, \ell=0-100$, for changing $A=10^{-1}, 10^{-2}$ and constant $\rho=10^{-1}$. It can be seen from the figure that, the BER performance approximately unchanged when changing the average number of impulses $A$, because when noise sources $\ell$ increases, $A^{\ell}$ will approach to zero and that leads to unchanged in the variance $\sigma_{A}^{2}$ and hence in $\sigma_{\beta}^{2}$. Thus, the system performance is unchanged.

The running process time of the simulated program for the conventional T-COFDM system is higher than the proposed T-COFDM system due to an additional calculations in the threshold optimization for each value of $\frac{E_{b}}{N_{0}}$. In addition to more running process time in the receiver side where the LLRs are computed based on Gaussian distribution which makes the conventional decoder iterates many times until detected the transmitted code word or until reached the maximum number of iterations. The running process time shown in Table I is calculated based on Fig. 4-a as reference of maximum transmitted frame is 50. In all cases, calculations have been made using a MATLAB R2018a simulation program in a personal laptop with an Intel Core $\mathrm{i} 7$ processor at $2.9 \mathrm{GHz}, 16 \mathrm{~GB}$ of RAM and $128 \mathrm{~GB}$ SSD using a 64-bit operating system.

The BER performance of QPSK modulation can be represented as a two orthogonal BPSK modulation. Therefore, due 


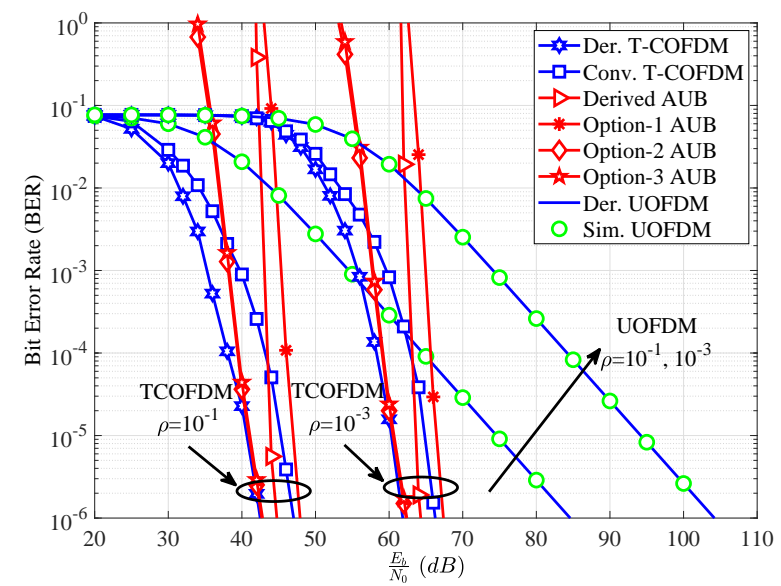

(a) Combined BI noise and IN with $A=10^{-2}$ and $\rho=10^{-1}, 10^{-3}$.

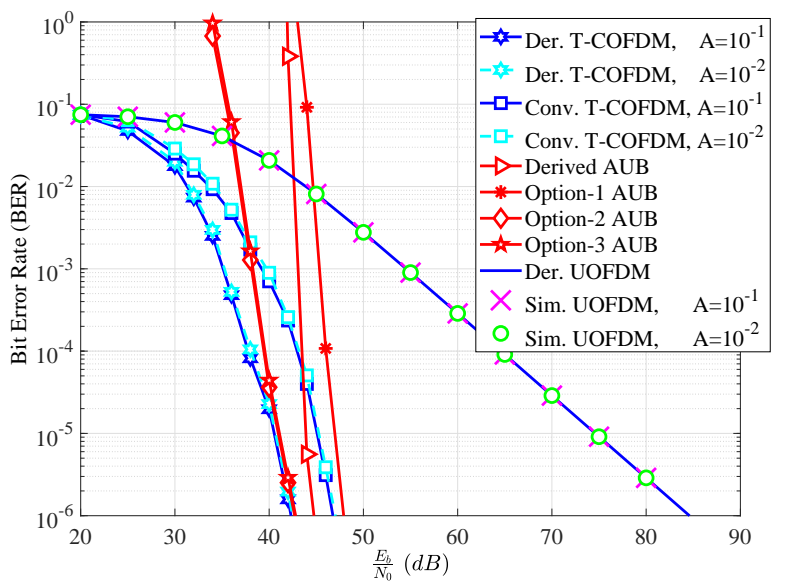

(b) Combined BI noise and IN with $A=10^{-1}, 10^{-2}$ and $\rho=10^{-1}$.

Fig. 4. Performance of proposed T-COFDM system versus conventional T-COFDM system utilizing 8192-QAM constellation over PLC with log-normal channel gain.

to the orthogonality, the real and imaginary components will not interfere each other, hence the BER performance of the QPSK modulation is identical to that of the BPSK modulation. Therefore, we have been simulated the BER performance of the proposed T-COFDM system and C-COFDM system by utilizing the QPSK modulation only. To prove the validity of the proposed T-COFDM system, Fig. 5 demonstrate the BER performance the proposed versus convolutional coded-OFDM (C-COFDM) system by utilizing QPSK modulation. The BER performance are investigated over the same channel parameters of Fig. 4-a. The proposed T-COFDM system outperforms the C-COFDM system over all $\frac{E_{b}}{N_{0}}$ and can achieve a BER $10^{-5}$ at $\frac{E_{b}}{N_{0}}=17.6 \mathrm{~dB}$ and $\frac{E_{b}}{N_{0}}=33.3 \mathrm{~dB}$. In contrast, the C-COFDM system required an additional margin of $29.4 \mathrm{~dB}$ and $33.1 \mathrm{~dB}$ to achieves the same BER performance at $47 \mathrm{~dB}$ and $66.4 \mathrm{~dB}$. This can be found on page 2 and bellow the Fig. 1, and is replicated below for convenience.

We proceed now to compare the complexity of convolutional codes versus TCs. Shannon's theorem indicates the BER performance of the coded system can be improved by using long block code for all practical purposes. Unfortunately, it may not be possible to build the convolutional decoder using optimal Viterbi decoding algorithm for long block length, i.e., (long memory or constraint length) to achieve the desired level of the BER performance. Moreover, the decoder complexity increases exponentially with the code length which introducing large processing latency. Therefore, parallel concatenation RSC codes or TCs is a very useful technique offering excellent BER performance close to Shannon's capacity. The two or more RSC codes of relatively constraint length achieved a lower complexity and which makes the iterative soft input soft output (SISO) decoder can be practically constructed with lower complexity. Thus effectively decomposing the problem of the decoding of a long block length in the case of the convolutional decoder.

Moreover, we proceed now to compare the power consumption of both systems by Considering the same noise environments of Fig. 4-a and by utilizing a QPSK constellation. To achieve a BER of $10^{-5}$, the proposed system needs a $\frac{E_{b}}{N_{0}}$ of approximately $17.6 \mathrm{~dB}$ and $33.3 \mathrm{~dB}$. In contrast, the convolutional-COFDM (C-COFDM) system required an additional margin power of 29.4 $\mathrm{dB}$ and $33.1 \mathrm{~dB}$ to achieve the same BER performance for the derived system at $47 \mathrm{~dB}$ and $66.4 \mathrm{~dB}$ as shown in Fig. 5. This 


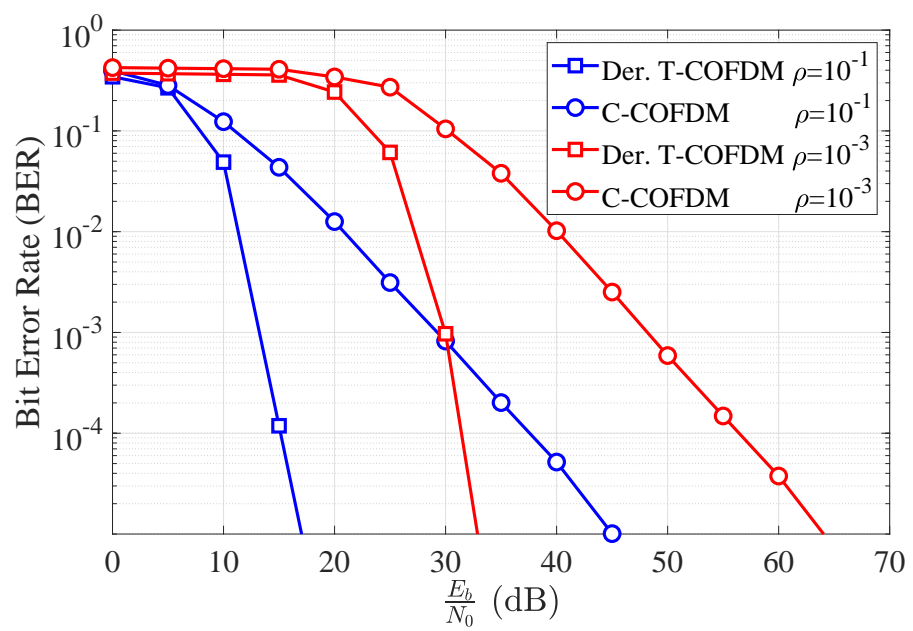

Fig. 5. Performance of proposed T-COFDM system versus C-COFDM system utilizing QPSK constellation over PLC channel with log-normal channel gain in the presence of combined BI with $m=0.7$ and IN with $A=0.01$ for $\rho=10^{-1}$ and $10^{-3}$.

can be found on page 2 and bellow Fig. 1, and is replicated below for convenience.

\section{CONCLUSION}

In this paper, the performance of T-COFDM has been improved by utilizing the LLRs computed from derived noise PDFs at the ZF equalizer output over the PLC channel. The noise distributions are derived at the ZF equalizer output for different scenarios, such as the Nakagami- $m$ BI noise, IN modelled by using MCAIN model and their combination. The proposed receiver has been verified by using Monte-Carlo simulations by utilizing 8192-QAM. Monte-Carlo simulation shows the proposed T-COFDM system utilizes derived PDFs outperforms the conventional receiver utilizes clipping impulsive noise mitigation method based on ML derived thresholds and LLRs computed based on the Gaussian distribution over PLC channel with log-normal gains in presence of different scenarios of non-Gaussian noise. Moreover, the average upper-bounds derived for the proposed T-COFDM system is bounded within less than $1 \mathrm{~dB}$ to Monte-Carlo simulations over PLC channel in the presence of different scenarios of individual and combined BI noise and IN.

\section{REFERENCES}

[1] A. Majumder, J. C. J. "Power line communications", IEEE Potentials, 23(4), pp. 4-8, (Oct 2004).

[2] J. Anatory, N. Theethayi. Broadband Power-line Communications Systems: Theory \& Applications, (wit press, 2010).

[3] M. Zimmermann, K. Dostert. "A multipath model for the powerline channel", IEEE Trans. on Commun., 50(4), pp. 553-559, (Apr. 2002).

[4] M. Zimmermann, K. Dostert. "Analysis and modeling of impulsive noise in broad-band powerline communications", IEEE Trans. Electromagn. Compat., 44(1), pp. 249-258, (2002).

[5] H. Meng, Y. L. Guan, S. Chen. "Modeling and analysis of noise effects on broadband power-line communications", IEEE Trans. Power Del., 20(2), pp. 630-637, (April 2005).

[6] Y. Kim, S. Choi, H. M. Oh. "Closed-form expression of nakagami-like background noise in power-line channel", IEEE Trans. Power Del., 23(3), pp. 1410-1412, (July 2008).

[7] D. Fertonani, G. Colavolpe. "On reliable communications over channels impaired by bursty impulse noise", IEEE Trans. on Commun., 57(7), pp. 2024-2030, (July 2009).

[8] M. Ghosh. "Analysis of the effect of impulse noise on multicarrier and single carrier QAM systems", IEEE Trans. on commun., 44(2), pp. 145-147, (Feb. 1996).

[9] J. G. Proakis. "Digital communications. 2008", McGraw-Hill, New York.

[10] S. Zhidkov. "Analysis and comparison of several simple impulsive noise mitigation schemes for OFDM receivers", IEEE Trans. on Commun., 56(1), pp. 5-9, (January 2008).

[11] H. A. Latchman, S. Katar, L. Yonge, S. Gavette. Homeplug AV and IEEE 1901: A Handbook for PLC Designers and Users, (John Wiley \& Sons, 2013).

[12] B. Edmonston. "Comparison of turbo-convolutional codes and turbo product codes for QPSK-64QAM channels", IEEE 802.16 Broadband Wireless Access Working Group.

[13] B. Tahir, S. Schwarz, M. Rupp. "BER comparison between Convolutional, Turbo, LDPC, and polar codes", Telecommunications (ICT), 2017 24th International Conference on, pp. 1-7, (IEEE, 2017).

[14] W. E. Ryan, et al. "An introduction to LDPC codes", , (2004).

[15] T. K. Moon. "Error correction coding", Mathematical Methods and Algorithms. Jhon Wiley and Son, (2005).

[16] H. Nakagawa, D. Umehara, S. Denno, Y. Morihiro. "A decoding for low density parity check codes over impulsive noise channels", Power Line Communs. and Its Appls., International Symposium on, pp. 85-89, (2005).

[17] D. Umehara, H. Yamaguchi, Y. Morihiro. "Turbo decoding in impulsive noise environment", Global Telecommun. Conf., 2004. GLOBECOM '04. IEEE, volume 1, pp. 194-198 Vol.1, (2004).

[18] H.-M. Oh, Y.-J. Park, S. Choi, J.-J. Lee, K.-C. Whang. "Mitigation of performance degradation by impulsive noise in LDPC Coded OFDM system", 2006 IEEE International Symposium on Power Line Communs. and Its Appls., pp. 331-336, (2006).

[19] V. Dimanche, A. Goupil, L. Clavier, G. Gelle. "On detection method for soft iterative decoding in the presence of impulsive interference", IEEE Commun. Lett., 18(6), pp. 945-948, (2014). 
[20] W. Gu, L. Clavier. "Decoding metric study for turbo codes in very impulsive environment", IEEE Commun. Lett., 16(2), pp. 256-258, (2012).

[21] J. Rebelatto, R. Souza, M. Pellenz. "On the performance of turbo codes in the presence of typical power line asynchronous impulsive noise", Networks, 2005. Jointly held with the 2005 IEEE 7th Malaysia International Conference on Communication., 2005 13th IEEE International Conference on, volume 2, pp. 5-pp, (IEEE, 2005).

[22] G. A. Al-Rubaye, C. C. Tsimenidis, M. Johnston. "Improved performance of TC-OFDM-PLNC for PLCs using exact derived impulsive noise PDFs", Communications Workshops (ICC Workshops), 2017 IEEE International Conference on, pp. 1271-1276, (IEEE, 2017).

[23] C. Berrou, A. Glavieux, P. Thitimajshima. "Near shannon limit error-correcting coding and decoding: Turbo-codes. 1", Communications, 1993. ICC '93 Geneva. Technical Program, Conference Record, IEEE International Conference on, volume 2, pp. 1064-1070 vol.2, (1993).

[24] R. Pacalet, M. J. G. Pina. "Full-Reconfigurable Interleaver Architecture for High-performance SDR applications", (SDR'10), pp. 509-514, (2010).

[25] S. Sesia, M. Baker, I. Toufik. LTE-the UMTS long term evolution: from theory to practice, (John Wiley \& Sons, 2011).

[26] M. Mirahmadi, A. Al-Dweik, A. Shami. "BER reduction of OFDM based broadband communication systems over multipath channels with impulsive noise", IEEE Trans. Commun., 61(11), pp. 4602-4615, (Nov. 2013).

[27] S. Galli. "A novel approach to the statistical modeling of wireline channels", IEEE Transactions on Communications, 59(5), pp. 1332-1345, (May 2011).

[28] I. C. Papaleonidopoulos, C. N. Capsalis, C. G. Karagiannopoulos, N. J. Theodorou. "Statistical analysis and simulation of indoor single-phase low voltage power-line communication channels on the basis of multipath propagation", IEEE Trans. Consum. Electron., 49(1), pp. 89-99, (Feb 2003).

[29] S. Guzelgoz, H. B. Celebi, H. Arslan. "Statistical characterization of the paths in multipath plc channels", IEEE Trans. Power Del., 26(1), pp. 181-187, (Jan 2011).

[30] D. Middleton. "Canonical and quasi-canonical probability models of class a interference”, IEEE Trans. Electromagn. Compat., EMC-25(2), pp. 76-106, (1983).

[31] S.Miyamoto, M. Katayama, N. Morinaga. "Performance analysis of QAM systems under class A impulsive noise environment", IEEE Trans. Electromagn. Compat, 37(2), pp. 260-267, (May 1995).

[32] A. Papoulis, S. U. Pillai. Probability, random variables, and stochastic processes, (Tata McGraw-Hill Education, 2002).

[33] A. Jeffrey, D. Zwillinger. Table of integrals, series, and products, (Academic Press, 2007).

[34] G. A. Al-Rubaye, C. C. Tsimenidis, M. Johnston. "LDPC-COFDM for PLC in Non-Gaussian Noise Using LLRs Derived from Effective Noise PDFs", accepted in IET on commun., 10.1049/iet-com.2017.0265, https://doi.org/10.1049/iet-com.2017.0265, (2017).

[35] P. Robertson, E. Villebrun, P. Hoeher. "A comparison of optimal and sub-optimal MAP decoding algorithms operating in the log domain", Communications, 1995. ICC'95 Seattle,'Gateway to Globalization', 1995 IEEE International Conference on, volume 2, pp. 1009-1013, (IEEE, 1995).

[36] M. K. Simon. Probability distributions involving Gaussian random variables: A handbook for engineers and scientists, (Springer Science \& Business Media, 2007).

[37] S. Benedetto, G. Montorsi. "Unveiling turbo codes: some results on parallel concatenated coding schemes", IEEE Trans. Inf. Theory, 42(2), pp. 409-428, (Mar 1996).

[38] E. K. Hall, S. G. Wilson. "Design and analysis of turbo codes on rayleigh fading channels", IEEE J. Sel. Areas Commun., 16(2), pp. 160-174, (Feb 1998).

[39] A. Spaulding. "Optimum reception in an impulsive interference environment (ph.d. thesis abstr.)", IEEE Trans. Inf. Theory, 21(6), pp. 721-721, (November 1975).

[40] G. Ndo, P. Siohan, M. H. Hamon. "An adaptive procedure for impulsive noise mitigation over power line channels", Proc. IEEE Global Telecommun. Conf. (GLOBECOM), pp. 1-6, (2009). 